\title{
TE WAVES AT A NONLINEAR INTERFACE IN KERR MEDIA
}

\author{
M. WABIA ${ }^{a}$ AND G. ŻEGLIŃSKI ${ }^{b}$ \\ ${ }^{a}$ Institute of Physics, Technical University of Szczecin \\ Al. Piastów 48, 70-310 Szczecin, Poland \\ ${ }^{b}$ Department of Optical Telecommunication and Photonics \\ Technical University of Szczecin, 26 Kwietnia 10, 71-062 Szczecin, Poland

\begin{abstract}
In this work a new type of solution for TE field on a plane interface of two nonabsorbing, optically self-focusing, nonlinear Kerr-type media has been presented. Numerical results are obtained for the dispersion equation and propagation constants of the field modes. The power guided by TE waves in the nonlinear interface was found and graphically illustrated.
\end{abstract} \\ PACS numbers: $42.65 .-\mathrm{k}$
}

\section{Introduction}

The electromagnetic field propagating at a boundary between different media exhibits a number of interesting properties which are the subject of several experimental and theoretical investigations.

In the case of linear media this field can be represented generally by different types of waves such as the lateral, surface or leaky ones [1-5].

In the nonlinear case papers of many authors have directed attention on a possibility of existence of the nonlinear surface waves. The earliest theoretical study of the TE polarized surface waves was carried out by Litvak and Mironov [6]. Recent studies [7-16] give actually a complete theory, determining properties and conditions of existence of the nonlinear surface waves. The modes are interesting because, as it is known, the TE polarized surface waves cannot exit on the planar interface between two linear dielectric media.

The problem of the existence of other than the nonlinear surface waves at the boundary between two nonlinear media has not yet, however, been satisfactorily investigated. On the basis of Kaplan's papers [7, 8] we know that when an electromagnetic plane wave is transmitted through a nonlinear interface, in some special cases inhomogeneous nonlinear waves perpendicular to the interface with a variable amplitude can be excited. Hence, the surface wave is not the only solution of the electromagnetic boundary problem as in the case of linear/nonlinear interface. 
The main purpose and the topic of this paper is to prove that the electromagnetic boundary problem in a nonlinear interface is more complicated than it is presented in the existing literature on this subject. The nonlinear interface can guide also the electromagnetic waves other than the surface waves with vanishing amplitude on both sides of the interface.

\section{The formulation of the problem}

Two semi-infinite regions, $x>0$ and $x<0$, separated by a plane interface $x=0$ will be considered, respectively. Each of the regions contains a uniaxial nonlinear medium of the Kerr type described by a dielectric tensor $j \widehat{\varepsilon}(j=1,2)$

$$
\dot{j} \widehat{\varepsilon}=\left(\begin{array}{ccc}
j_{\varepsilon_{11}} & 0 & 0 \\
0 & j_{\varepsilon_{22}} & 0 \\
0 & 0 & j_{\varepsilon_{33}}
\end{array}\right) .
$$

The geometry of the problem is shown in Fig. 1. We consider the stationary nonlinear TE waves propagating in the $z$ direction with $z$-dependence in the standard form $[7-16]$

$$
{ }^{j} E_{y}(x, z, t)={ }^{j} E(x) \exp \left[\mathrm{i}\left(k_{0} h z-\omega t\right)\right]+\text { c.c. },
$$

where $k_{0}$ is the free space wave number, $h$ is the mode index and $\omega$ is the angular frequency.

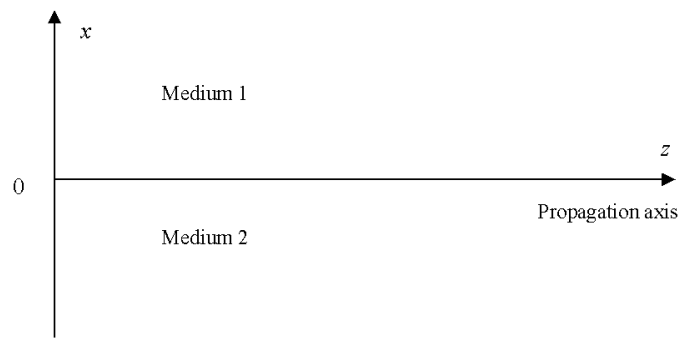

Fig. 1. Geometry of considered problem.

From Maxwell's equations, for TE guided waves travelling down the $z$ axis, the basic equations are

$$
\begin{aligned}
& k_{0} h^{j} E(x)=-\omega \mu_{0}{ }^{j} H_{x}(x), \\
& \frac{\mathrm{d}^{j} E(x)}{\mathrm{d} x}=\mathrm{i} \omega \mu_{0}{ }^{j} H_{z}(x), \\
& \frac{\mathrm{d}^{j} H_{z}(x)}{\mathrm{d} x}=\mathrm{i} \omega \varepsilon_{0}{ }^{j^{j} \varepsilon_{22}}{ }^{j} E(x),
\end{aligned}
$$

where $\varepsilon_{0}$ and $\mu_{0}$ are the permittivity and the permeability of free space, respectively. Eliminating ${ }^{j} H_{x}(x)$ and ${ }^{j} H_{z}(x)$ from the set of Eqs. (3-5) one obtains the wave equation for $j$-th region

$$
\frac{\mathrm{d}^{2}{ }^{j} E}{\mathrm{~d} x^{2}}-k_{0}^{2}\left(h^{2}-{ }^{j} \varepsilon_{22}\right)^{j} E=0,
$$


where ${ }^{j} E={ }^{j} E_{y}(x)$. Assuming the Kerr law nonlinearity, in which the ${ }^{j} \varepsilon_{22}$ is given by

$$
j_{\varepsilon_{22}}=j_{\varepsilon_{1}}+\left.\left.\alpha_{j}\right|^{j} E\right|^{2}
$$

we have

$$
\frac{\mathrm{d}^{2}{ }^{j} E}{\mathrm{~d} x^{2}}-k_{0}^{2}\left(h^{2}-{ }^{j} \varepsilon_{22}\right)^{j} E+k_{0}^{2} \alpha_{j}|E|^{2}{ }^{j} E=0 .
$$

In the latter expressions ${ }^{j} \varepsilon_{1}$ denotes the linear permittivity, $\alpha_{1}$ is the nonlinear coefficient of $x>0\left({ }^{1} \varepsilon_{1}, \alpha_{1}\right)$ and $x<0\left({ }^{2} \varepsilon_{1}, \alpha_{2}\right)$ regions, respectively. yields

For a self-focussing medium, $\alpha_{j}>0$, and the first integration of Eq. (8)

$$
\left(\frac{\mathrm{d}^{j} E}{\mathrm{~d} x}\right)^{2}-k_{0}^{2}\left[\left(h^{2}-{ }^{j_{\varepsilon_{1}}}\right){ }^{j} E_{2}+\frac{\alpha_{j}}{2}{ }^{{ }^{j}} E^{4}\right]=C_{j},
$$

where $C_{j}$ is an integration constant.

In the previous studies referred to in this introduction, the electromagnetic field problem at a nonlinear interface is reduced only to the surface modes, which do not carry any energy along the $x$-axis. For this reason $C_{j}$ then must be set equal to zero constant for both sides of the semi-infinite nonlinear media $\left(C_{1}=0, C_{2}=0\right)$.

This paper focuses on investigation of solutions other than the surface waves. Our strategy assumes that in both half-spaces of the nonlinear interface there can also exist modes for which $C_{j} \neq 0$ on one or both sides of the interface. Such prediction can be made on the basis of analysis of the phenomenon in the linear case, where apart from the surface modes other waves appear (leaky waves and lateral waves).

In the presently investigated problem we seek such solutions that would be oscillating and periodic in the region $x<0$ and vanishing in the region $x>0$, therefore we put $C_{2}>0$, for $x<0$ and $C_{1}=0$, for $x>0$. In the theory of propagation of the electromagnetic field in a semi-infinite medium, the conditions at infinity must be defined. Since there are no sources inside the half-spaces $x>0$ and $x<0$, the following conditions have to be satisfied:

$$
\begin{aligned}
& E(x) \rightarrow 0 \text { for } x \rightarrow+\infty, \\
& E(x) \rightarrow \text { const } \equiv E_{\infty}(x) \geq 0 \text { for } x \rightarrow-\infty,
\end{aligned}
$$

which are, essentially, the Sommerfeld radiation conditions (the absence of the backward-travelling wave).

\section{The solution of the equations}

$$
\text { 3.1. The region } 1, x>0, \alpha_{1}>0
$$

According to the assumptions made in ch. 2, $C_{1}=0$, and the first integral of Eq. (8) is

$$
\left(\frac{\mathrm{d}^{1} E}{\mathrm{~d} x}\right)^{2}-k_{0}^{2}\left(h^{2}-{ }^{1} \varepsilon_{1}\right){ }^{1} E^{2}+\frac{k_{0}^{2} \alpha_{1}}{2}{ }^{1} E^{4}=0 .
$$


This equation has an exact solution

$$
{ }^{1} E=p_{1}\left\{\operatorname{ch}\left[q_{1} k_{0}\left(x-x_{01}\right)\right]\right\}^{-1},
$$

where

$$
\begin{aligned}
& p_{1}=\left[2\left(h^{2}-1_{\varepsilon_{1}}\right) / \alpha_{1}\right]^{1 / 2}, \\
& q_{1}=\left(h^{2}-{ }^{1} \varepsilon_{1}\right)^{1 / 2},
\end{aligned}
$$

$x_{01}$ is the location of the maximum of the electrical field strength.

$$
\text { 3.2. The region } 2, x<0, \alpha_{2}>0
$$

The first integral of Eq. (8) is presently given by

$$
\left(\frac{\mathrm{d}^{2} E}{\mathrm{~d} x}\right)^{2}-k_{0}^{2}\left(h^{2}-{ }^{2} \varepsilon_{1}\right)^{2} E^{2}+\frac{k_{0}^{2} \alpha_{2}}{2}{ }^{2} E^{4}=C_{2},
$$

where $C_{2}>0$ is an integration constant.

Then the general solution of Eq. (16) can be expressed as

$$
{ }^{2} E(x)=p_{2} \operatorname{cn}\left[q_{2} k_{0}\left(x-x_{02}\right) / m\right],
$$

where $\mathrm{cn}$ is a specific Jacobi elliptic function, $x_{02}$ is a second integration constant. Parameters $p_{2}, q_{2}$, and the modulus $m$ are given by

$$
\begin{aligned}
& p_{2}^{2}=\frac{h^{2}-{ }^{2} \varepsilon_{1}+\left[\left(h^{2}-{ }^{2} \varepsilon_{1}\right)^{2}+2 \alpha_{2} C_{2} / k_{0}^{2}\right]^{1 / 2}}{\alpha_{2}}, \\
& q_{2}^{2}=\left[\left(h^{2}-{ }^{2} \varepsilon_{1}\right)^{2}+2 \alpha_{2} C_{2} / k_{0}^{2}\right]^{1 / 2} \\
& m=\frac{\alpha_{2} p_{2}^{2}}{2 q_{2}^{2}}
\end{aligned}
$$

\section{The continuity conditions and the integration constant}

The boundary conditions at the interface $x=0$ require the continuity of the tangential components of $E(x)$ and $H_{z}(x)$. Therefore we have, respectively

$$
\begin{aligned}
& { }^{1} E(0)={ }^{2} E(0), \\
& \left.\frac{\mathrm{d}^{1} E(x)}{\mathrm{d} x}\right|_{x=0}=\left.\frac{\mathrm{d}^{2} E(x)}{\mathrm{d} x}\right|_{x=0} .
\end{aligned}
$$

Using Eqs. (12), (16), and (22) one can find easily the $C_{2}$ constant

$$
C_{2}=k_{0}^{2}\left({ }^{2} \varepsilon_{1}-{ }^{1} \varepsilon_{1}\right) E_{0}^{2}+\left(k_{0}^{2} / 2\right)\left(\alpha_{1}-\alpha_{2}\right) E_{0}^{4},
$$

where $E_{0}$ is the value of the electric field at the interface $x=0$. 


\section{The dispersion equation}

The continuity conditions (21) and (22) for the tangential component give the relations

and

$$
p_{1}\left[\operatorname{ch}\left(k_{0} q_{1} x_{01}\right)\right]^{-1}=p_{2} \operatorname{cn}\left(k_{0} q_{2} x_{02}\right),
$$

$$
p_{1} q_{1} \operatorname{th}\left(k_{0} q_{1} x_{01}\right)\left[\operatorname{ch}\left(k_{0} q_{1} x_{01}\right)\right]^{-1}=p_{2} q_{2} \operatorname{sn}\left(k_{0} q_{2} x_{02}\right) \operatorname{dn}\left(k_{0} q_{2} x_{02}\right) .
$$

Equations (24) and (25) yield the general dispersion equation

$$
q_{1} \operatorname{th}\left(k_{0} q_{1} x_{01}\right) \operatorname{cn}\left(k_{0} q_{2} x_{02}\right)=q_{2} \operatorname{sn}\left(k_{0} q_{2} x_{02}\right) \operatorname{dn}\left(k_{0} q_{2} x_{02}\right) \text {. }
$$

Because the electric field on the interface plane at $x=0$ is now given by

$$
\begin{aligned}
& E_{0}=p_{1} \operatorname{ch}^{-1}\left(q_{1} k_{0} x_{01}\right), \\
& E_{0}=p_{2} \operatorname{cn}\left(q_{2} k_{0} x_{02}\right),
\end{aligned}
$$

where it follows that the dispersion equation (26) can be written in the form

$$
p_{2} q_{1}\left(p_{1}^{2}-E_{0}^{2}\right)=p_{1} q_{2}\left(p_{2}^{2}-E_{0}^{2}\right)\left[p_{2}^{2}-m^{2}\left(p_{2}^{2}-E_{0}^{2}\right)\right]^{1 / 2} .
$$

After some simple transformations the last equation assumes an elementary form of the algebraic equation for the mode index $h$

$$
2\left(h^{2}-{ }^{2} \varepsilon_{1}\right)^{2}\left(A^{2}-B^{2}\right)+4\left(h^{2}-{ }^{2} \varepsilon_{1}\right) B C+C\left(A^{2}-2 C\right)=0,
$$

where

$$
\begin{aligned}
& A=2\left({ }^{2} \varepsilon_{1}-{ }^{1} \varepsilon_{1}\right)+\left(5 \alpha_{1}-4 \alpha_{2}\right) E_{0}^{2}, \\
& B=2\left({ }^{2} \varepsilon_{1}-{ }^{1} \varepsilon_{1}\right)+\left(\alpha_{1}-2 \alpha_{2}\right) E_{0}^{2}, \\
& C=2 \alpha_{2}\left[2\left({ }^{2} \varepsilon_{1}-{ }^{1} \varepsilon_{1}\right) E_{0}^{2}+\left(\alpha_{1}-\alpha_{2}\right) E_{0}^{4}\right] .
\end{aligned}
$$

\section{Analysis of the dispersion equation}

The dispersion equation is easily to solve, and we find

$$
h= \pm\left\{{ }^{2} \varepsilon_{1}-\frac{2 B C \mp \sqrt{2 A^{2} C\left[2 C-\left(A^{2}-B^{2}\right)\right]}}{2\left(A^{2}-B^{2}\right)}\right\}^{1 / 2} .
$$

Equation (34) determines the propagation constants $h(\omega)$ representing the waves propagating along the nonlinear interface. As seen with (34), (31-33), the values $h$ depend on the guided wave power by interface. It is interesting to note that the values (34) can be real or complex. Two kinds of wave modes can exist for this reason. The configuration considered here is ideally lossless. From the literature it is known that in such a case the real values $h$ must correspond to the guided surface waves, the complex values $h$ correspond to the guided leaky waves.

When

$$
2 A^{2} C\left[2 C-\left(A^{2}-B^{2}\right)\right]<0
$$

the roots of the dispersion equation become complex otherwise they become real or imaginary. 


\subsection{Complex roots}

a)

The simple consideration demonstrates that the inequality (35) is satisfied if

$$
C>0
$$

$$
2 C-\left(A^{2}-B^{2}\right)<0
$$

${ }^{2} \varepsilon_{1}>{ }^{1} \varepsilon_{1}, \quad \alpha_{2}>\frac{3 \alpha_{1}}{2}$

$$
\frac{2\left({ }^{2} \varepsilon_{1}-{ }^{1} \varepsilon_{1}\right)}{\alpha_{2}-\alpha_{1}}>E_{0}^{2}>\frac{2\left({ }^{2} \varepsilon_{1}-{ }^{1} \varepsilon_{1}\right)}{2 \alpha_{2}-3 \alpha_{1}},
$$

or b)

$$
C>0
$$

$$
2 C-\left(A^{2}-B^{2}\right)<0
$$

${ }^{1} \varepsilon_{1}>{ }^{2} \varepsilon_{1}, \quad \alpha_{1}>\alpha_{2}$,

$E_{0}^{2}>\frac{2\left({ }^{1} \varepsilon_{1}-{ }^{2} \varepsilon_{1}\right)}{\alpha_{1}-\alpha_{2}}$,

and c)

$$
C<0 \text {, }
$$

$$
2 C-\left(A^{2}-B^{2}\right)>0,
$$

$$
{ }^{1} \varepsilon_{1}>{ }^{2} \varepsilon_{1}, \quad \alpha_{1}>\frac{2 \alpha_{2}}{3},
$$

$$
E_{0}^{2}<\frac{2\left({ }^{1} \varepsilon_{1}-{ }^{2} \varepsilon_{1}\right)}{3 \alpha_{1}-2 \alpha_{2}} .
$$

\subsection{Real roots}

The condition of the real roots of the dispersion equation existence results from the inequality

$$
C\left[2 C-\left(A^{2}-B^{2}\right)\right]>0 .
$$

It is easy to prove that such roots will exist in the case when the below conditions are satisfied:

a)

$$
C>0
$$




$$
\begin{aligned}
& 2 C-\left(A^{2}-B^{2}\right)>0, \\
& { }^{2} \varepsilon_{1}>{ }^{1} \varepsilon_{1}, \quad \alpha_{2}>\frac{3 \alpha_{2}}{2}, \\
& E_{0}^{2}<\frac{2\left({ }^{2} \varepsilon_{1}-{ }^{1} \varepsilon_{1}\right)}{2 \alpha_{1}-3 \alpha_{2}},
\end{aligned}
$$

and $b$ )

$$
C<0 \text {, }
$$

$$
2 C-\left(A^{2}-B^{2}\right)<0
$$

$$
{ }^{2} \varepsilon_{1}>{ }^{1} \varepsilon_{1}, \quad \alpha_{2}>\alpha_{1},
$$

$$
E_{0}^{2}<\frac{2\left({ }^{2} \varepsilon_{1}-{ }^{1} \varepsilon_{1}\right)}{\alpha_{2}-\alpha_{1}}
$$

or c)

$$
C<0
$$

$$
2 C-\left(A^{2}-B^{2}\right)<0
$$

$$
{ }^{1} \varepsilon_{1}>{ }^{2} \varepsilon_{1}, \quad \alpha_{1}>\alpha_{2},
$$

$$
\frac{2\left({ }^{1} \varepsilon_{1}-{ }^{2} \varepsilon_{1}\right)}{\alpha_{1}-\alpha_{2}}>E_{0}^{2}>\frac{2\left({ }^{1} \varepsilon_{1}-{ }^{2} \varepsilon_{1}\right)}{3 \alpha_{1}-2 \alpha_{2}} .
$$

As one can say, the solutions of the dispersion equation are always comprised in three intervals but in a special way:

- if ${ }^{1} \varepsilon_{1}-{ }^{2} \varepsilon_{1}>0$ and $\alpha_{1}-\alpha_{2}>0$, two intervals of complex solutions and one interval of real solution exist;

- if ${ }^{1} \varepsilon_{1}-\varepsilon_{1}<0$ and $\alpha_{1}-\alpha_{2}<0$, two intervals of real solutions and one interval of complex solutions exist.

These conclusions are illustrated in Figs. 2 and 3.

Presuming that the first medium $(x>0)$ is liquid crystal MBBA whose refractive index has its linear and nonlinear parts of the values [13] of $n_{01}=1.55$ and $n_{21}=10^{-9} \mathrm{~m}^{2} / \mathrm{W}$, and the second medium $(x<0)$ is crystal YAG with the adequate values of the refractive index [13] as $n_{02}=1.83$ and $n_{22}=3 \times 10^{-9} \mathrm{~m}^{2} / \mathrm{W}$, four real solutions of the dispersion equation are obtained, appropriately: $h=$ \pm 1.71 and $h= \pm 2.72$. These values were obtained for $E_{0}=10^{5} \mathrm{~V} / \mathrm{m}$. 


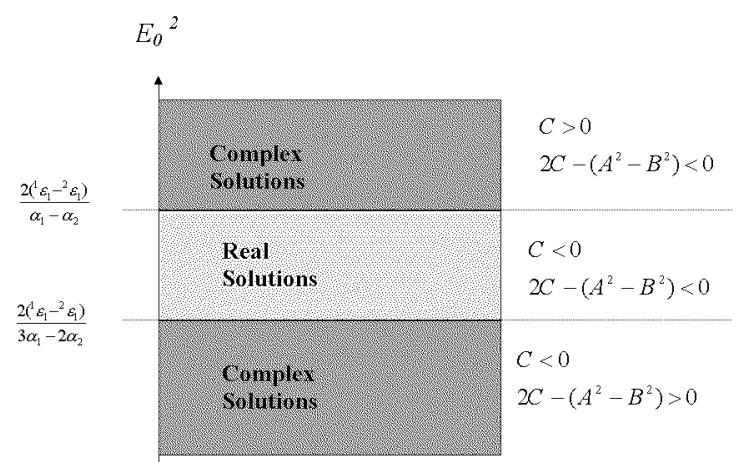

Fig. 2. The solutions of the dispersion equation when ${ }^{1} \varepsilon_{1}-{ }^{2} \varepsilon_{1}>0, \alpha_{1}-\alpha_{2}>0$.

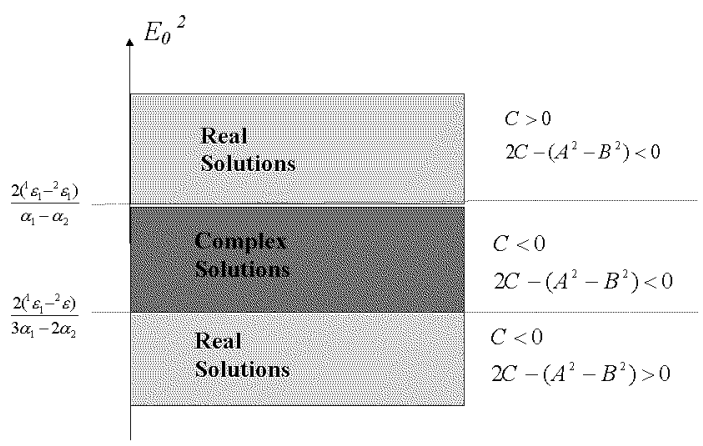

Fig. 3. The solution of the dispersion equation when ${ }^{2} \varepsilon_{1}-{ }^{1} \varepsilon_{1}>0, \alpha_{2}-\alpha_{1}>0$.

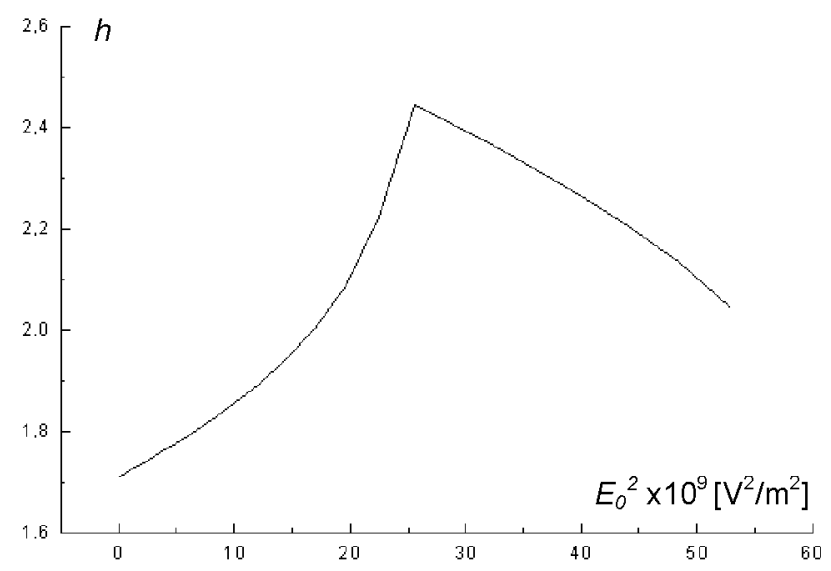

Fig. 4. Propagation constant $h$ dependence on the strength of electric field $E_{0}$ on the interface. 
The dependence of the propagation constant $h$ from the value of electrical field $E_{0}$ on the nonlinear interface for the above configuration is illustrated in Fig. 4.

Assuming that the propagation constant is of calculated value $h=1.71$, it is possible to make a graphical illustration of the electrical field profile at the nonlinear interface, adequate to the solution of this boundary problem. The profile is presented in Fig. 5 .

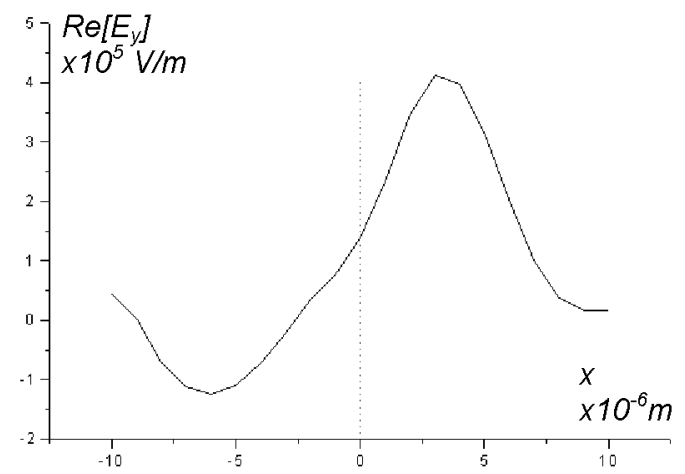

Fig. 5. Profile of TE electric field on the nonlinear interface.

The electrical TE field of the wave guided by the nonlinear interface has its maximum in the region of $x>0$. In the region of $x<0$ the wave amplitude oscillates between positive and negative values which results in the average value equal to zero in the period of oscillations.

\section{The power in regions and the entire power}

The power flow for a harmonic field is described by the Poynting vector

$$
\langle\boldsymbol{S}\rangle=\frac{1}{2} \operatorname{Re}\left[\boldsymbol{E} \times \boldsymbol{H}^{*}\right] .
$$

For the TE field $E_{y}, H_{x}$, and $H_{z}$ we have

$$
\langle\boldsymbol{S}\rangle=\frac{1}{2} \operatorname{Re}\left[\mathrm{i}\left(E_{y} H_{z}^{*}\right)-\boldsymbol{k}\left(E_{y} H_{x}^{*}\right)\right]
$$

Hence, the power flow $\mathrm{P}$ transported by the waves in the $z$ direction is

$$
\begin{aligned}
P= & \int_{-\infty}^{+\infty} k\langle\boldsymbol{S}\rangle \mathrm{d} x=P_{1}+P_{2}= \\
& \frac{k_{0} h}{2 \omega \mu_{0}} \int_{0}^{\infty}\left|E_{y}(x)\right|^{2} \mathrm{~d} x+\frac{k_{0} h}{2 \omega \mu_{0}} \int_{0}^{T}\left|E_{y}(x)\right|^{2} \mathrm{~d} x .
\end{aligned}
$$

The entire power is the sum of the powers $P_{1}$ and $P_{2}$ from the regions 1 and 2 . 


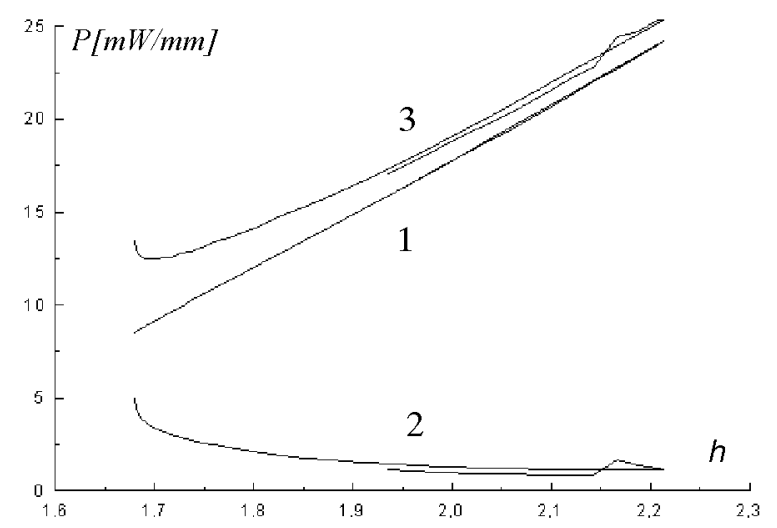

Fig. 6. Guided power dependence on the propagation constant; 1 - power in the region 1,2 - power in the region 2,3 - total power.

The power guided in the regions 1 and 2 as well as the entire power depending on the propagation constant $h$ are illustrated in Fig. 6.

The illustrations 5 and 6 shows that the nonlinear interface can guide TE waves with specific and new characteristics. These waves, while propagating along the interface in the direction perpendicular to the interface, are of variable amplitude which vanishes in the region 1 and periodically oscillates in the region 2 . These waves can be guided for some propagation constants $h$ and over the certain threshold of power. One can also see that the distinct minimum of power exists, for which the mode of the field can be guided. Moreover, two propagation constants $h$ (upper and lower) exist, by which the guided modes are cut.

\section{Flow of power guided in the direction perpendicular to the interface}

For a better characterisation of the wave described in the previous paragraphs, the flow of power and the power guided by such wave in the direction perpendicular to the interface should be calculated.

For this purpose the more general form of $\mathrm{TE}$ wave is considered, taking into account also its dependence upon the coordinate $x$, i.e. the field with the component $E_{y}(x, z, t)$

$$
E_{y}(x, z, t)=E(x) \exp \left[\mathrm{i}\left(k_{0} h z+\phi(x)-\omega t\right)\right] .
$$

The average flow of power in the direction perpendicular to the interface is defined by the component of the expression (62) and given as

$$
\langle\boldsymbol{S}\rangle_{x}=\frac{1}{2} \operatorname{Re}\left[E_{y}(x, z) H_{z}^{*}(x, z)\right] .
$$

Using (64) and (4) it is easy to prove that this component is of the shape

$$
\langle\boldsymbol{S}\rangle_{x}=\frac{1}{2 \omega \mu_{0}}\left|E_{y}(x)\right|^{2} \frac{\mathrm{d} \phi(x)}{\mathrm{d} x} .
$$


The field TE on the nonlinear interface, investigated in this paper, is described by the expression (2), for which

$$
\frac{\mathrm{d} \phi(x)}{\mathrm{d} x}=0 .
$$

The above expression indicates that the average flow of power in the direction perpendicular to the interface, which is created by the analysed wave on the interface, is equal to zero

$$
\langle\boldsymbol{S}\rangle_{x}=0 .
$$

Such behaviour is specific for surface waves. The standard surface waves on the interface were investigated by many researchers [10-14]. The characteristics of such waves are perfectly known. Apart from the characteristic that the flow of power guided in the direction perpendicular to the interface is equal to zero, the other characteristic is the fast vanishing of the amplitude in the mentioned direction, which can be observed on both sides of the interface. In the above context, the here-investigated wave can be qualified as the surface wave or quasi-surface wave because of the non-typical behaviour of amplitude.

Comparison of the expressions (66) and (67) indicates for one more characteristic of wave (2) guided by the nonlinear interface: the average flow of power guided by the wave perpendicular to the interface direction is always equal to zero, irrespective of the character of amplitude changes in this direction.

\section{Discussion of the achieved results}

The discussion of the achieved results and their interpretation, being the topic of this chapter, will be more clear if some results taken from literature are mentioned first:

1. Linear interface (of two linear media) cannot guide the wave of TE type $[10-16]$.

2. In the case of nonlinear interface and focusing medium the surface wave of TE type can be guided, with the amplitude vanishing on both sides of the interface. Nonlinearity creates then its specific waveguide guiding the wave of the soliton type $[6,16]$.

In the context of the above, the results achieved in this paper allow us to define the following conclusions:

a) The solutions (13) and (17) on nonlinear interface can be matched in such a way that the boundary conditions on the interface are satisfied as well as the condition of radiation in infinity. The further effect is that the electromagnetic wave with TE polarisation can be guided on interface, and penetrates with its amplitude in the characteristic way, the nonlinear media surrounding the interface.

b) The amplitude of the wave in surrounding media is given by the function vanishing in the direction perpendicular to the interface for $x>0$ and oscillating periodically for $x<0$. 
c) The average flow of power guided by the wave perpendicular to the interface direction is equal to zero, irrespective of the amplitude changes in this direction.

d) The above-mentioned electromagnetic field follows the known conservation of energy flux in the nonlinear medium [13, 14]. From the point of view of power flow, the wave behaves like the nonlinear surface wave, but at the same time it shows another surface wave shape of amplitude. From that reason the analysed TE wave on the interface can be considered as the surface or quasi-surface wave.

e) A certain critical minimum and maximum of guided power exist, below and over which the wave of such type cannot be excited.

f) The waves with the amplitude variable perpendicularly to the interface are not a new phenomenon in the problem of nonlinear waves. On the basis of Kaplan's papers $[7,8]$ we know that in special cases inhomogeneous nonlinear waves with the amplitude variable perpendicularly to the interface can be excited on the interface. They are described by the amplitude functions

$$
A^{2}=A_{\infty}^{2} \pm 2 B^{2}\left\{\begin{array}{c}
\sin h^{-2} \\
\cos h^{-2}
\end{array}\right\}\left(B k_{0} z+C\right),
$$

where $B$ and $C$ are certain constants, $A_{\infty}$ is the value of amplitude in infinity. These waves are called longitudinally inhomogeneous travelling waves (LITW).

g) The formal analysis of the dispersion equation (34) allows the propagation constant $h$ to be complex. From the physical point of view this case corresponds to the inhomogeneous wave with the amplitude which is vanishing towards the direction of propagation, i.e. towards the $z$ direction. The case of lossless media is connected with the leak of wave guided inside the media surrounding the interface, like in a partial reflection of wave on the interface. That case will not be however the topic of the detailed analysis in this paper.

\section{References}

[1] T. Tamir, Optik 36, 209 (1972).

[2] T. Tamir, Optik 37, 204 (1973).

[3] T. Tamir, Optik 38, 269 (1973).

[4] M. Wabia, Acta Phys. Pol. A 78, 861 (1990).

[5] M. Wabia, Acta Phys. Pol. A 78, 873 (1990).

[6] A.G. Litvak, A. Mironov, Izv. UVZ Radiofiz. 11, 1911 (1968).

[7] A.E. Kaplan, IEEE J. Quantum Electron. QE-17, 336 (1981).

[8] A.E. Kaplan, Sov. Phys. IETP 45, 896 (1977).

[9] W.J. Tomlinson, Opt. Lett. 5, B23 (1980).

[10] A.A. Maradudin, Optical and Acoustic Waves in Solids - Modern Topics, World Scientific Pub., Singapore 1983, Ch. 2. 
[11] G.I. Stegeman, C.T. Seaton, J. Ariyasu, R.F. Wallis, A.A. Maradudin, J. Appl. Phys. 58, 2453 (1985).

[12] T.P. Shen, A.A. Maradudin, G.I. Stegeman, J. Opt. Soc. Am. B5, 1391 (1988).

[13] A.D. Boardman, P.E. Egan, F. Lederer, U. Langbein, D. Michalache, Nonlinear Surface Electromagnetic Phenomena, North-Holland, Amsterdam 1991, Ch. 2.

[14] A.D. Boardman, P.E. Egan, T. Twardowski, M. Wilkins, Nonlinear Waves in Solid State Physics, Plenum Press, New York 1992, Ch. 1.

[15] E. Wright, G.I. Stegeman, Anisotropic and Nonlinear Optical Waveguides, Elsevier Science Pub., New York 1992, p. 117.

[16] J.V. Moloney, Guided Wave Nonlinear Optics, Kluwer Academic Publishers, Boston 1992, p. 201. 